\title{
Improved diagnostic accuracy of malignant neck lumps by a simple BMVC staining assay $\dagger$
}

\author{
Li-Jen Liao, ${ }^{a b}$ Chi-Chih Kang, ${ }^{c d}$ I-Shiow Jan, ${ }^{e}$ Huei-Chin Chen, ${ }^{a}$ Chiung-Lin Wang, ${ }^{c}$ Pei-Jen Lou ${ }^{* a}$ \\ and Ta-Chau Chang*c
}

\author{
Received 19th August 2008, Accepted 9th January 2009 \\ First published as an Advance Article on the web 24th January 2009 \\ DOI: $10.1039 / \mathrm{b814417f}$
}

\begin{abstract}
A handheld device based on fluorescence of 3,6-bis(1-methyl-4-vinylpyridinium)carbazole diiodide (BMVC) staining was established for the rapid, point-of-care screening of cancer cells (see Chang and co-workers, Analyst, 2007, 132, 745). Offering instant screening of cancer at low cost, here we apply this simple assay in clinical tests on fine needle aspirates of neck masses from 114 outpatients (115 specimens). The diagnostic accuracy of this simple method alone is $c a .80 \%(80 / 99)$. The combination of the BMVC test and the fine needle aspiration (FNA) cytology reduced the non-diagnosis from 17 cases in FNA cytology to 6 cases in the combined method. Moreover, an algorithm is proposed to improve the diagnostic accuracy of malignant neck lumps up to nearly $100 \%$.
\end{abstract}

\section{Introduction}

Cancer is a universal disease and remains a major cause of death. Despite substantial progress in understanding the fundamental mechanisms of carcinogenesis and searching for new therapeutic approaches for cancer treatment, the development of innovative cancer screening and diagnostic methods with high accuracy and low cost is still a high priority for current practice. Discovering the presence of cancer cells in the early stages could greatly improve treatment options and effectively reduce cancer mortality.

Fine needle aspiration (FNA) is a useful diagnostic technique that provides simple, easy, and cost-effective means for the diagnosis of accessible lesions with almost no side effects for the patients. ${ }^{1}$ Generally, FNA cytology has a high overall diagnostic accuracy with minimal complications. ${ }^{1,2}$ Thus, FNA cytology has been used as a first-line screening tool for the head and neck, ${ }^{3}$ thyroid, ${ }^{4}$ and breast, ${ }^{5}$ etc., lesions. However, interpretation of the smearing of aspirations is sometimes difficult to master even for specialists. ${ }^{6}$ It would be better if one can develop an adjuvant method to FNA cytology for the more effective diagnosis of cancer cells.

\footnotetext{
${ }^{a}$ Department of Otolaryngology, National Taiwan University Hospital and National Taiwan University College of Medicine, Taipei, Taiwan, Republic of China.E-mail:pjlou@ntu.edu.tw

${ }^{b}$ Department of Otolaryngology, Far Eastern Memorial Hospital, Taipei, Taiwan, Republic of China

${ }^{c}$ Institute of Atomic and Molecular Sciences, Academia Sinica, P.O. Box 23-166, Taipei, Taiwan 106, Republic of China. E-mail: tcchang@po. iams.sinica.edu.tw

${ }^{d}$ Taiwan International Graduate Program and Department of Chemistry, National Tsing-Hua University and Academia Sinica, Taiwan, Republic of China

${ }^{e}$ Department of Laboratory Medicine, National Taiwan University Hospital and National Taiwan University College of Medicine, Taipei, Taiwan, Republic of China

$\dagger$ Electronic supplementary information (ESI) available: results of diagnosis of malignant neck masses and results of FNA cytology and BMVC test. See DOI: 10.1039/b814417f
}

Recently, a simple handheld device based on the fluorescence analysis of 3,6-bis(1-methyl-4-vinylpyridinium)carbazole diiodide (BMVC) stained cells was established to instantly differentiate cancer cells from normal cells for non-specialists at a very low cost. ${ }^{7,8}$ To verify if the BMVC test is a useful tool in clinical cancer diagnosis, we have measured the sensitivity, specificity, positive predictive value (PPV), and negative predictive value (NPV) of the BMVC test and compared them with that of the FNA cytology for palpable neck masses. Comparison of results from these two methods allows us to propose a simple combinational assay that significantly improves the diagnostic accuracy of malignant neck lumps. It should be emphasized that the BMVC test does not require additional puncture for the patient.

\section{Experimental}

\subsection{A simple device of BMVC test for the screening of cancer cells}

The synthesis of BMVC from 3,6-dibromo-9H-carbazole through the Heck reaction and methylation has been described elsewhere. 9 A handheld device includes a light emitting diode, a $470 \mathrm{~nm}$ band pass filter for exciting the BMVC, a $520 \mathrm{~nm}$ long pass filter for transmitting the emission of the BMVC, and a digital camera to record the images for further data analysis. ${ }^{7}$ The BMVC emission could also be visualized by the human eye without using a detector. We note that the handheld device is compact and can be operated with minimal training. The device does not have any sophisticated equipment and can be easily constructed in-house.

\subsection{In vitro study}

A cell line of MRC-5 human lung fibroblasts was purchased from ATCC (American Type Culture Collection) and grown under their complete growth media. A CL1-0 human lung cancer cell line was provided by Professor C. T. Chen of the National Taiwan University. Cells were grown on a T25 flask and harvested 
by trypsin EDTA. The harvested CL1-0 human lung cancer cells and MRC-5 lung fibroblasts were independently stained by $2 \mu \mathrm{M}$ $\mathrm{BMVC}$ or $10 \mathrm{nM}$ acridine orange (AO) in the growth medium as a function of incubation time. The samples were centrifuged at $2500 \mathrm{rpm}$ for $5 \mathrm{~min}$ to remove the medium and then resuspended in PBS. The cell pellets, resuspended in a $300 \mu \mathrm{L}$ PBS with a cell density of $c a .1 \times 10^{6}$ cells $/ \mathrm{mL}$, were further studied by using flow cytometry. In addition, a ca. $2 \mu \mathrm{L}$ cell suspension in an Eppendorf tube incubated with $2.0 \mu \mathrm{M}$ BMVC for $10 \mathrm{~min}$ was examined by using our simple device.

\subsection{A pilot ex vivo study of fine needle aspiration of palpable neck lumps}

A 23-gauge needle was introduced into the neck lumps. Cellular material was aspirated and smeared onto slides for cyto-pathological diagnosis. The residual cells in the needle and syringe were washed out by using DMEM culture medium, and then centrifuged at $1000 \mathrm{rpm}$ for $5 \mathrm{~min}$. The cells were resuspended with fresh culture medium and equivalent volumes of cells were dropped onto three different slides and stained by trypan blue (TB), AO, and BMVC, respectively. Observation for TB under an optical microscope was applied to confirm cell viability. Using our screening device, AO staining was used to estimate the total number of cells under screening and BMVC staining was assessed for screening of the cancer cells. The study protocol was approved by the Institutional Review Board at the National Taiwan University Hospital (NTUH, No. 9561709041) and informed consent was obtained from all participants.

\section{Results and discussion}

\subsection{Distinct properties of BMVC for cancer screening}

We have illustrated that the fluorescence yield of BMVC increases by two orders of magnitude upon interacting with DNA. ${ }^{9,10}$ In addition, epi-fluorescence images showed that several bright fluorescence spots of BMVC appeared in the nucleus of cancer cells, but were rarely observed in the nucleus of normal cells. ${ }^{6}$ Accordingly, the much brighter fluorescence of BMVC in the cancer cells than that in the normal cells can be applied to differentiate cancerous from normal cells. ${ }^{7}$

To confirm whether there are sufficient samples examined under the BMVC test, we have used AO to estimate the number of cells. This is because AO can rapidly stain both cancer cells and normal cells. ${ }^{11}$ Moreover, we have optimized the concentration of $\mathrm{AO}$ as an additional criterion for differentiating the fluorescence of BMVC in cancer cells from normal cells. Fig. 1(A) shows the fluorescence intensities of $2.0 \mu \mathrm{M}$ BMVC and $10 \mathrm{nM}$ AO incubated with human lung CL1-0 cancer cells and MRC-5 normal cells as a function of time measured by flow cytometry. It is found that the BMVC fluorescence in the cells of CL1-0 is ca. 5 times larger than that of MRC-5 at 10 min incubation time. We further found that the fluorescence intensity of $\mathrm{AO}$ in cells is maximized at around $10 \mathrm{~min}$. By carefully adjusting the concentration of $\mathrm{AO}$ to $10 \mathrm{nM}$, a fluorescence intensity similar to that from $2.0 \mu \mathrm{M}$ BMVC incubated with cancer cells can be detected in either cancer or normal cells. Using the handheld device, ${ }^{7}$ Fig. 1(B) showed the fluorescence emission of CL1-0 cancer cells and MRC-5 normal cells incubated with

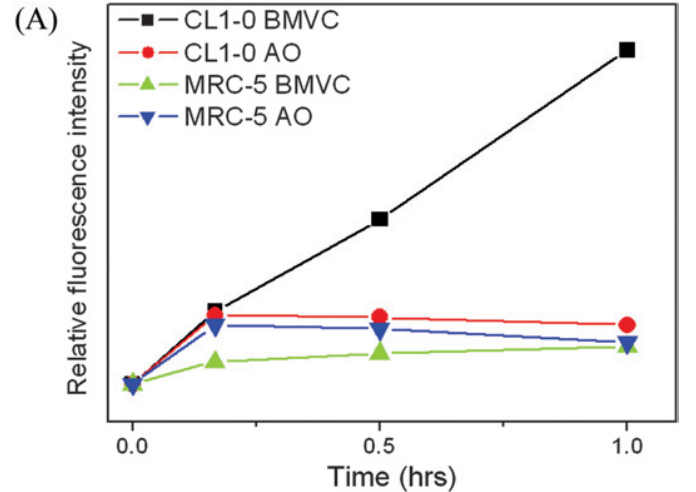

(B)

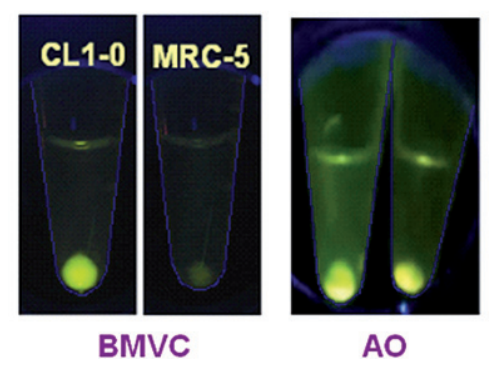

Fig. 1 (A) Fluorescence intensities of $2 \mu \mathrm{M} \mathrm{BMVC}$ and $10 \mathrm{nM}$ AO as a function of incubated time in CL1-0 and MRC-5 cells measured by flow cytometry. (B) Distinguishing of CL1-0 cancer cells from MRC-5 normal cells in the tips of Eppendorf tubes by BMVC under the excitation of an LED light source. AO is applied to further stain the cells for reference.

$2.0 \mu \mathrm{M}$ of $\mathrm{BMVC}$ for $10 \mathrm{~min}$. It is found that significantly brighter fluorescence of $\mathrm{BMVC}$ is indeed detected in the cancer cells than in the normal cells. We then added $10 \mathrm{nM}$ AO to both of them and confirmed that there were sufficient cells in both Eppendorf tubes, as also shown in Fig. 1(B). This additional criterion is very useful for some suspected cases.

\subsection{A pilot study of fine needle aspiration cytology for palpable neck masses}

The success of using the handheld device for differentiating cancer cells from normal cells in vitro prompted us to test this simple assay in a clinical test. A pilot study of 114 outpatients (61 females and 53 males) from the Department of Otolaryngology at NTUH between June 2006 and October 2007 has been examined to evaluate the feasibility of the BMVC test in real cytological samples. Could it be an adjuvant tool for FNA cytology to improve the diagnostic accuracy of malignant neck lumps? Fig. 2(A) shows the fluorescence images of a sample stained by AO and BMVC. The sample from the residual cells of a fine needle aspirate was collected from a 29 -year-old female. The weak fluorescence of BMVC suggested that the test is negative. The FNA cytology and pathology revealed a benign submandibular tumor. Fig. 2(B) shows the images of both stains of a sample collected from a 69 -year-old man. The much more obvious fluorescence from BMVC suggested that the test was positive. The diagnosis was hypopharyngeal cancer with neck metastasis. Among 114 patients with 115 specimens, the BMVCtested results suggested 46 positive, 53 negative and 16 nondiagnostic cases. 


\section{(A)}
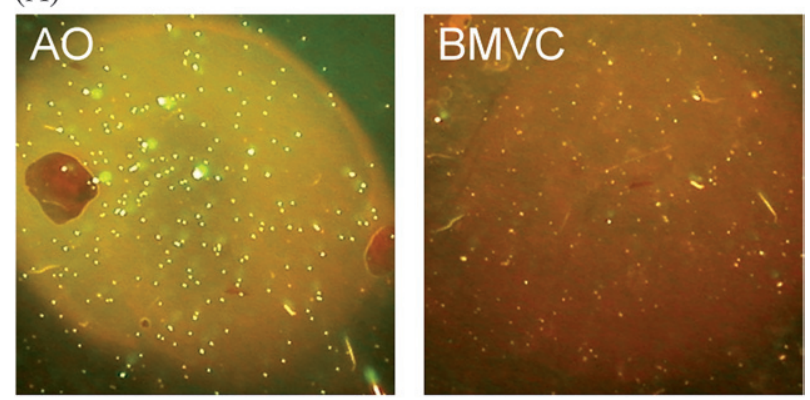

(B)
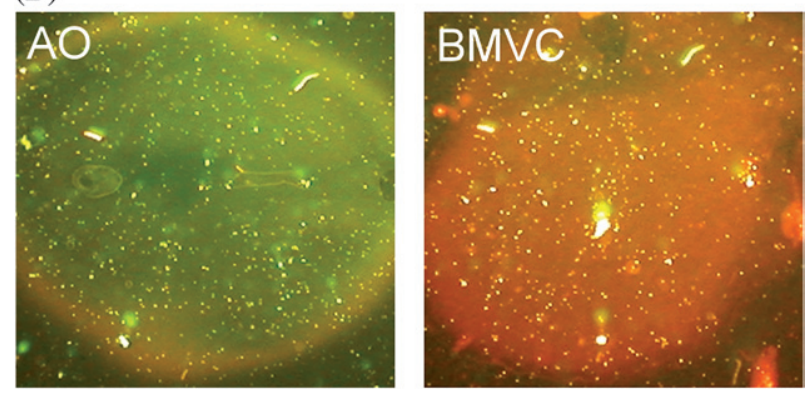

Fig. 2 (A) A 29-year-old female presented with a submandibular tumor. The FNA cytology and pathology revealed a benign mixed tumor. The BMVC test was negative. The enhanced fluorescence of BMVC is less than AO. (B) A 69-year-old male complained of hoarseness and neck mass for one month. The diagnosis was hypopharyngeal cancer with neck metastasis. The BMVC test was positive. The enhanced fluorescence of $\mathrm{BMVC}$ is much obvious than $\mathrm{AO}$.

It is essential to compare our BMVC results to the FNA cytological results and the final results of diagnosis. The final diagnoses revealed that there were $42(36.5 \%)$ malignant tumors and $73(63.5 \%)$ benign tumors. The median age of the patients was 47 years (range 10-89 years). Final diagnosis for malignancy was made from pathological examination of the surgical specimen $(n=30)$, or FNA cytology $(n=12)$. The diagnosis of benign processes was dependent upon negative pathological exam or cytological result with at least 3 months of follow-up. Neck masses that were not enlarged in size or disappeared during the follow-up period were considered as benign. The list of the origin of these malignant neck masses and the results of FNA cytology and BMVC test can be found in Supplementary 1 (ESI $\dagger$ ). Among the 42 malignant neck masses, most of the cases were metastatic nodes from primary tumors within the head and neck region. Metastatic nodes from cancers out of the head and neck region such as lung cancer and thymic cancer were also encountered. Nine patients were proved to be hematological malignancies such as multiple myeloma or lymphoma based on histological examination of the excised neck masses. The list of the diagnosis of 73 benign neck masses and the results of FNA cytology and BMVC test can be found in Supplementary 2 (ESI $\dagger$ ). Among the 73 benign neck masses, benign lymphoid hyperplasia was the most frequently encountered diagnosis, followed by thyroid and salivary gland tumors.

Using FNA cytology, 28 specimens were suggested to be malignant. All of them were finally proved to be malignant in origin, indicating that the PPV was $100 \%(28 / 28)$ by FNA cytology. There were 70 specimens classified as negative for malignancy; however, 7 cases were finally diagnosed as malignancy and BMVC tests were all positive. The NPV was $c a .90 \%$ (63/70) by FNA cytology. Although the diagnostic accuracy of FNA cytology for malignant neck lumps is $c a .93 \%$, there were 17 specimens classified as non-diagnostic due to insufficient cellularity or crushing artifacts in the cytology. Among these 17 specimens, the BMVC test revealed 8 specimens as positive and 6 of the 8 specimens were finally diagnosed as malignant in origin.

The BMVC test achieved a PPV of $c a .70 \%(32 / 46)$ and a NPV of $c a .90 \%$ (48/53) with 16 non-diagnostic cases among the 115 specimens from neck lumps. Since sufficient vital cells are required for the BMVC test, a small amount of cells trapped in the needle and syringe in some cases may lead to false negative and non-diagnostic results. Unexpectedly, benign cells in some clinical samples were positively stained that resulted in false positive. The false positive may be due to autofluorescence of RBC (red blood cells) and BMVC fluorescence upon interaction with some contaminants in the aspirates or DNA in dead cells, which are not found in the screening of cell lines. Nevertheless, the BMVC test is shown to be a simple and inexpensive tool that does not need a specialist to conduct the test and review the result. The $c a .80 \%$ diagnostic accuracy of the BMVC test in this ex vivo study supports that the BMVC test is a useful tool for the point-of-care screening of malignant neck lumps in clinical practices. In addition, the problem of false positive results that were encountered in real cytological samples may provide invaluable information for the development of small molecules for cancer screening.

\subsection{An algorithm proposed for improving diagnostic accuracy}

High sensitivity and specificity is the key for the development of a molecular analysis in cancer diagnosis. Table 1 summarizes the results of FNA cytology, the BMVC test, and the combined method for the diagnosis of malignant neck lumps. Here the combined method classifies as positive when either the cytology or BMVC test diagnosed as positive. The combined method is regarded as negative or non-diagnostic when both the cytology and BMVC tests show negative or non-diagnostic. It is found that the BMVC test has a sensitivity of $86.5 \%$, a specificity of $77.4 \%$, and an overall accuracy of $80.0 \%$. Although the BMVC test is not sufficient to replace the FNA cytology that has

Table 1 FNA cytology, BMVC test, and combined method in the diagnosis of malignant neck masses ${ }^{a}$

\begin{tabular}{|c|c|c|c|}
\hline & \multicolumn{3}{|c|}{ No./total no. $(95 \%$ CI $)$} \\
\hline & FNA cytology & BMVC test & $\begin{array}{l}\text { Combined } \\
\text { method }\end{array}$ \\
\hline Non-diagnosis & $17 / 115(14.8 \pm 7)$ & $16 / 115(13.9 \pm 6)$ & $6 / 115(5.2 \pm 4)$ \\
\hline Sensitivity & $28 / 35(80.0 \pm 14)$ & $32 / 37(86.5 \pm 11)$ & $41 / 41(100.0 \pm 0)$ \\
\hline Specificity & $63 / 63(100.0 \pm 0)$ & $48 / 62(77.4 \pm 11)$ & $54 / 68(79.4 \pm 10)$ \\
\hline Accuracy & $91 / 98(92.9 \pm 5)$ & $80 / 99(80.8 \pm 8)$ & $95 / 109(87.2 \pm 6)$ \\
\hline PPV & $28 / 28(100.0 \pm 0)$ & $32 / 46(69.6 \pm 14)$ & $41 / 55(74.5 \pm 12)$ \\
\hline NPV & $63 / 70(90.0 \pm 7)$ & $48 / 53(90.6 \pm 8)$ & $54 / 54(100.0 \pm 0)$ \\
\hline
\end{tabular}




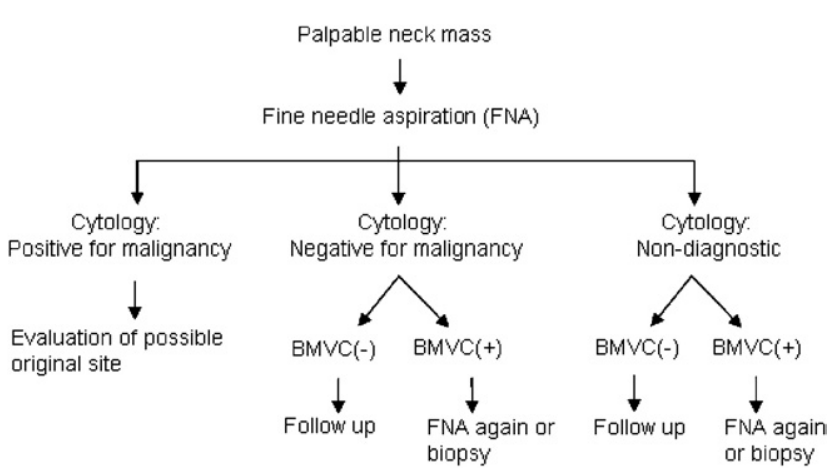

Scheme 1 Neck mass FNA algorithm.

a sensitivity of $80.0 \%$, a specificity of $100.0 \%$, and an overall accuracy of $92.9 \%$, the non-diagnostic rate was significantly reduced from $14.8 \%$ in FNA cytology to the $5.2 \%$ in the combined method. In addition, the sensitivity can be improved to $100 \%$ in the combined method. Here we take advantage of the higher specificity of the FNA cytology and the better sensitivity in the combined method to propose an algorithm for the effective screening of malignant neck lumps (Scheme 1).

We propose that in addition to the routine FNA procedure, the residual cells in the needle lumen should be examined by the $\mathrm{BMVC}$ test. This BMVC test does not require additional puncture for the patient and offers an instant diagnostic result of neck lumps. If the FNA cytology yields a positive result for malignancy, it is no doubt for the clinician to look for possible primary sites or arrange a biopsy for pathological confirmation. If the cytology result is negative while the BMVC test is positive, a repeated FNA cytology exam or open biopsy is suggested. On the other hand, if both FNA cytology and BMVC test results are negative, it is more reliable as a true negative and the patient only need to be followed up. In cases of sampling error or other causes that lead to a non-diagnostic FNA exam, which is quite common in clinical practice, the patient may only need to be followed up if the BMVC test is negative. Otherwise, a repeated FNA exam is suggested if the BMVC test is positive. Despite the 28 malignant cases examined by FNA cytology, there were still 27 positive cases screened by BMVC test. Without considering the 6 nondiagnostic cases in both assays, the repeated exam on 27 patients allows us to improve the overall accuracy to nearly $100 \%$, accordingly.

\subsection{Exciting changes for cancer detection}

'Accuracy' with no false positive as well as no false negative results is the ultimate goal for cancer diagnosis. An error as a false positive will cause great anxiety and unnecessary cost to the patient, and an error as a false negative will lead to miserable harm to the patient. Considering cancer screening, it is better to have an assay with the highest sensitivity for early detection and reducing the risk of missing the cancer. Here we have illustrated that the sensitivity of the BMVC test is $86.5 \%$ alone and can be improved to $100 \%$ for the patients with neck lumps while in combination with FNA cytology. Moreover, the proposed algorithm provides a promising assay to minimize not only the false negatives but also false positives for cancer screening. We believe that this combination can much improve cancer diagnosis of malignant neck lumps.

Besides the accuracy, many people dream of a simple device for instant diagnosis of cancer. In addition, the benefit of having an inexpensive screening is also a major goal for cancer diagnosis. ${ }^{12}$ This is particularly important in community health centers and the developing world. The BMVC test is an inexpensive point-of-care screening test that provides immediate results within $20 \mathrm{~min}$ and does not require additional puncture to the patient. Here the BMVC is simply an adjuvant tool to FNA cytology for cancer screening. There is no additional cost to the patient. Thus, we highly recommend that the BMVC test be added to the diagnostic protocol in FNA cytology for cancer screening. It is also of interest to apply the BMVC test to other FNA samples, such as breast, prostate, etc.

The success of the BMVC test is mainly due to two distinct properties of the fluorescence probe: significant increase of the fluorescence yield upon interaction with DNA and the large time lag of adhesion of BMVC to the nucleus between cancer cells and normal cells. ${ }^{7,8}$ However, the mechanism of the sensitivity of BMVC staining to cancer cells remains unraveled. Understanding the mechanism of the ligand sensitivity to cancer cells over normal cells is critical for the development of new fluorescence markers. It appears that further design of a better fluorescence probe to a specific cellular target is a promising direction for improving the accuracy of cancer diagnosis. Moreover, this simple assay can be applied to screening other diseases if one can find a molecular marker with the target specificity and fluorescence sensitivity to a specific disease.

\section{Acknowledgements}

This work was supported by Academia Sinica (AS-95-TP-AB2) and the National Science Council of the Republic of China (Grant NSC-96-2113-M001-024).

\section{References}

1 I. D. Buley and D. E. Roskell, Clin. Oncol., 2000, 12, 166-171.

2 B. D. Florentine, B. Staymates, M. Rabadi, J. Barstis and A. Black, Cancer, 2006, 107, 406-416.

3 R. G. Amedee and N. R. Dhurandhar, Laryngoscope, 2001, 111, $1551-1557$.

4 M. Xing, R. P. Tufano, A. P. Tufaro, S. Basaria, M. Ewertz, E. Rosenbaum, P. J. Byrne, J. Wang, D. Sidransky and P. W. Ladenson, J. Clin. Endocrinol. Metab., 2004, 89, 2867-2872.

5 P. Zagorianakou, S. Fiaccavento, N. Zagorianakou, G. Makrydimas, D. Stefanou and N. J. Agnantis, Eur. J. Gynaecol. Oncol., 2005, 26, 143-149.

6 D. C. Howlett, B. Harper, M. Quante, A. Berresford, M. Morley, J. Grant, K. Ramesar and S. Barnes, J. Laryngol. Otol., 2007, 121, 571-579.

7 C. C. Kang, C. C. Chang, T.-C. Chang, L. J. Liao, P. J. Lou, W. Xie and E. S. Yeung, Analyst, 2007, 132, 745-749.

8 C. C. Chang, I. C. Kuo, J. J. Lin, Y. C. Lu, C. T. Chen, H. T. Back, P. J. Lou and T.-C. Chang, Chem. Biodiversity, 2004, 1, 1377-1384.

9 C. C. Chang, J. Y. Wu and T.-C. Chang, J. Chin. Chem. Soc., 2003, 50, $185-188$.

10 C. C. Chang, J. Y. Wu, C. W. Chien, W. S. Wu, H. Liu, C. C. Kang, L. J. Yu and T.-C. Chang, Anal. Chem., 2003, 75, 6177-6183.

11 D. M. Neville and D. F. Bradley, Biochim. Biophys. Acta, 1961, 50, 397-399.

12 I. Borget, P. Vielh, S. Leboulleux, M. Allyn, S. Iacobelli, M. Schlumberger and G. de Pouvourville, Am. J. Clin. Pathol., 2008, 129, 763-771. 